\title{
Sialolitos en conductos y glándulas salivales. Revisión de literatura
}

\section{Sialoliths in ducts and salivary glands. Literature review}

\author{
Rebolledo Cobos $M^{*}$, Carbonell Muñoz Z**, Díaz Caballero A***
}

\section{RESUMEN}

La sialolitiasis es una afección que se produce por la obstrucción de una glándula salival o de su conducto excretor por la formación de concreciones calcáreas o sialolitos en el parénquima de los mismos. Existen teorías que afirman que los sialolitos en las glándulas y conductos salivales son originados por la mineralización de varios componentes como: cuerpos extraños, detritus celulares y microorganismos, depositándose inicialmente una matriz orgánica, probablemente de glucoproteínas, para luego posteriormente presentarse el deposito de material inorgánico que inicia su mineralización. Esta patología desencadena una serie de signos y síntomas hasta la obstrucción del conducto que no permite el paso de la saliva, lo que produce sintomatología dolorosa y tumefacción.

Existen otras entidades patológicas de glándulas salivales que pueden confundirse con sialolitiasis como sialoadenitis, hipertrofia maseterina, patologías relacionadas con la articulación temporomandibular, osteomielitis, mucocele, otras como quistes de retención mucoso, abscesos sublinguales y otras alteraciones del piso de la boca. Los métodos más comunes utilizados para el diagnóstico de sialolitiasis son la sialografía convencional, ecografía, resonancia magnética nuclear, tomografía asistida por computador, endoscopia, en ocasiones las radiografías laterales de cráneo y radiografías oclusales. Estos métodos diagnósticos son variables según las necesidades del paciente y accesibilidad, localización del sialolito, el tamaño del mismo y los signos y síntomas presente. El manejo de estas alteraciones incluye procedimientos no quirúrgicos y quirúrgicos invasivos o no que implican en ocasiones la eliminación de la glándula.

Palabras clave: Sialolitiasis, glándulas salivales, diagnóstico.

\section{SUMMARY}

Sialolithiasis is a clinical condition produced by a blockage of the salivary gland or excretory duct by the formation of calcareous concretions or sialoliths in the parenchyma of the same. There are theories that claim that the sialoliths in the salivary glands and ducts are caused by the mineralization of various components such as foreign bodies, cellular debris and microorganisms, originally deposited an organic matrix, probably of glycoproteins, and then subsequently submitted to the General Assembly Hall of inorganic material which began its mineralization. This condition triggers a series of signs and symptoms to duct obstruction that does not allow the passage of saliva, which causes pain and swelling.

There are other pathological salivary glands that can be confused with Sialolithiasis as sialadenitis, Masseteric hypertrophy, diseases related to the joint temporomandidular, osteomyelitis, mucocele, as other mucous

* Odontóloga Fundación Universitaria San Martín Sede Caribe. Residente de Estomatología y Cirugía Oral Universidad de Cartagena.

** Odontóloga Estomatóloga y Cirujana Oral. Universidad de Cartagena. Especialista en Estomatología y Cirugía Oral. Universidad de Cartagena. Profesora titular Universidad de Cartagena.

*** Odontólogo Universidad de Cartagena Especialista en Periodoncia Universidad Javeriana Magíster en educación Universidad del Norte. Candidato a Doctor en Ciencias Biomédicas Universidad de Cartagena. Profesor titular Universidad de Cartagena. 
retention cysts, abscesses and other disturbances of sublingual floor of the mouth. The most common methods used to diagnose Sialolithiasis are sialograph conventional ultrasound, magnetic resonance imaging, computer tomography, endoscopy, sometimes lateral radiographs of the skull and occlusal X-rays. These diagnostic methods are variable depending on the patient's needs and accessibility, sialolith location, the size of it and the signs and symptoms present. The management of these disorders include non-surgical and surgical procedures or non-invasive sometimes involving the removal of the gland.

Key words: Sialolithiasis, salivary glands, diagnosis.

Fecha de recepción: 19 de febrero 2009.

Aceptado para publicación: 25 de febrero 2009.

Rebolledo Cobos M, Carbonell Muñoz Z, Díaz Caballero A. Sialolitos en conductos y glándulas salivales. Revisión de literatura. Av. Odontoestomatol 2009; 25 (6): 311-317.

\section{INTRODUCCIÓN}

La sialolitiasis es una entidad patológica que consta de la obstrucción mecánica de la glándula salival o de su conducto excretor por formación de cálculos o sialolitos en el parénquima de los mismos, secundariamente puede producirse infección de la misma dando un cuadro se sialolitiasis crónica $(1,2)$. Estas formaciones calcáreas pueden ser únicas o múltiples y su forma puede variar entre ovalada o redondeada (3). La verdadera causa no se conoce con exactitud, pero hay varias hipótesis al respecto. Todas ellas concuerdan en que los sialolitos se forman por la mineralización de materiales diversos como: cuerpos extraños, células epiteliales descamadas y microorganismos. En algunas ocasiones primero se deposita una matriz orgánica, probablemente de glucoproteínas, sobre la que posteriormente se deposita el material inorgánico e inicia su mineralización (4). Algunas teorías sugieren que pueden deberse a la presencia de soluciones de continuidad en el conducto como traumatismos, que posiblemente dificultan el flujo salival y provocan estancamiento; otra sugiere que la saliva está hipersaturada con respecto a calcio y fósforo, esto es la causa principal de la formación del cálculo. Algunos autores opinan que puede ser ocasionado por infecciones bacterianas, virales, fúngicas, o a la irritación causada por cuerpos extraños como restos alimenticios acumulación de restos epiteliales con la posterior formación del cálculo $(5,6)$. Nahlieli et al, opinan que una evagina- ción intraductal puede ser el factor desencadenante de la sialolitiasis (7). En cualquier caso parece ser que la suma circunstancial de varias de estas causas son las que ponen en marcha el proceso de precipitación del fosfato tricálcico amorfo, que, una vez cristalizado y transformado en hidroxiapatita, dan a la formación del cálculo (6). Asimismo se señala que la terapia con medicamentos antihipertensivos o diuréticos que sean capaces de inhibir o disminuir la salivación y el hiperparatiroidismo secundario a insuficiencia renal crónica, podrían ser un factores predisponentes a padecer esta enfermedad (8).

Los sialolitos pueden aparecer a cualquier edad, pero el pico de máxima frecuencia se sitúa entre la cuarta y sexta décadas de la vida (9). La Sialolitiasis es muy rara en los niños, sin embargo existen casos reportados (6). Con respecto al sexo de los pacientes, en diferentes estudios sobre sialolitiasis se observa predominio en los varones en una razón de 2:1 (10).

Esta enfermedad representa el $11 \%$ de los casos de disfunción de las glándulas salivales. Es más frecuente en la glándula submaxilar en un (90\%), con la menor frecuencia aparece en la glándula parótida en un $(6 \%)$ y en muy raras ocasiones se le observa asociada a las glándulas sublinguales y las glándulas salivares menores en un $(2 \%)(2,11)$. Algunos autores observaron que se asocia más comúnmente al lado izquierdo y rara vez es bilateral, la afectación de la glándula parótida por un cálculo salival es más 
frecuente de forma unilateral, y localizado en el sistema ductal. El tamaño suele ser más pequeño que los sialolitos submandibulares y la mayoría son menores de $1 \mathrm{~cm}$ (12).

\section{Composición}

Los sialolitos están formados por dos tipos de componentes, un grupo de origen orgánico y otro inorgánico. Entre los principales constituyentes orgánicos se encuentran productos de la saliva (glucoproteínas y mucopolisacáridos), lípidos y detritus celulares. El componente inorgánico principal es el carbonato-apatito y no la hidroxiapatita acompañado de otras sales de calcio, además de diferentes tipos de fosfatos, magnesio, hierro, cobre y zinc. La mineralización de la matriz orgánica está favorecida por: 1) aumento del $\mathrm{pH}$ que permite la precipitación del fosfato de calcio de la saliva, 2) intensificación de la concentración de mucina en la saliva y su capacidad para transportar calcio y 3) alteración del medio iónico de la saliva (13). Los sialolitos son de tamaño y formas diferentes, con respecto al color por lo general se presentan de color amarillo o parduzco y su superficie puede ser lisa o irregular (6).

\section{SIGNOS Y SÍNTOMAS}

Los pacientes con sialolitiasis suelen no presentar dolor, pero cuando se presenta puede variar desde moderado hasta severo, por lo general se presenta antes, durante y después de las comidas, así como también al probar alimentos ácidos o salados debido a la estimulación del flujo salival, esta sintomatología se presenta en un $17 \%$ de los casos (5). La oclusión del conducto impide el paso de la saliva y el estancamiento provoca presión intraductal, lo que produce dolor y tumefacción. En algunas ocasiones el paciente no presenta síntomas notables y la única manifestación puede ser la presencia de una lesión tumoral de consistencia pétrea, que se palpa en el conducto o en el interior de la glándula. Es fundamental realizar una inspección cuidadosa, constatando la asimetría para establecer la presencia de un aumento de volumen, cantidad de flujo salival, además de realizar palpación cuidadosa de la glándula y zona ductal, buscando áreas nodulares o firmes, es aconsejable tomar una radiografía del área afectada para determinar la localización exacta del cálculo (7) (Fig. 1).

\section{DIAGNÓSTICOS DIFERENCIALES}

El diagnóstico diferencial de los agrandamientos bilaterales asintomáticos de la región parotídea incluyen las lesiones linfoepiteliales (síndrome de Mikulicz), síndrome de Sjögren, las sialoadenosis que acompañan al alcoholismo (14), la toma prolongada de diversos medicamentos (como el yodo y metales pesados) y al tumor de Whartin (15). Los agrandamientos bilaterales dolorosos pueden originarse tras radioterapia o pueden ser secundarios a una sialoadenitis vírica (incluyendo las paperas) cuando se acompañan de otros síntomas sistémicos. Entre los cuadros que cursan con inflamación facial difusa de la región parotídea, pero sin relación con las glándulas, debemos incluir la hipertrofia del músculo masetero, lesiones relacionadas con la articulación temporomandidular y la osteomielitis de la rama ascendente de la mandíbula. En cuanto a las entidades patológicas diferenciales de las glándulas salivales sublingual y submaxilar podemos encontrar quistes de retención mucosa como el mucocele, otras como la ránula, abscesos sublinguales y otras alteraciones inflamatorias e infecciosas del piso de la boca. Tiene importancia diferenciar los sialolitos de otras calcificaciones de los tejidos blandos, mientras algunos se

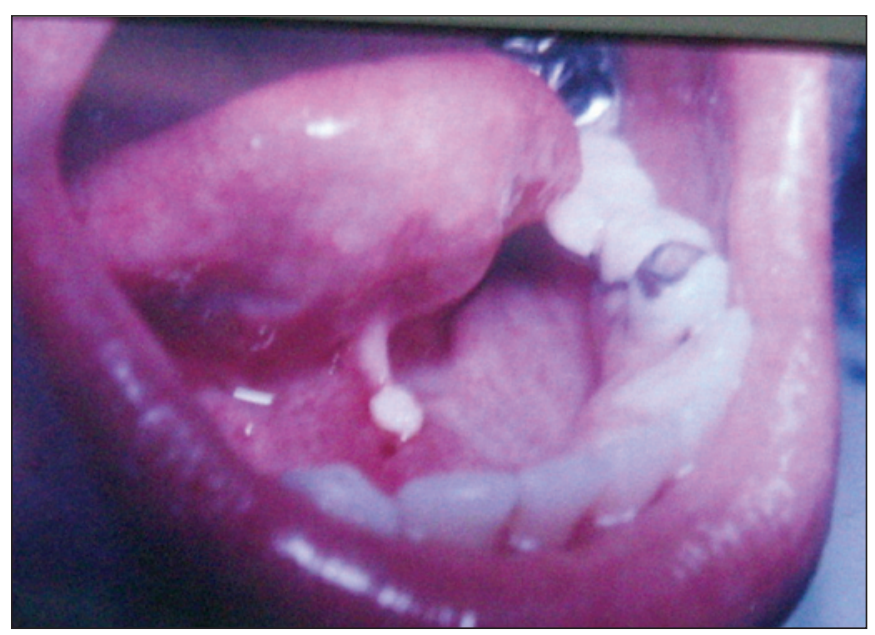

Fig. 1. Aspecto clínico de un sialolito en glándula sublingual. 
asocian con dolor y tumefacción en las glándulas salivares hay otras patologías semejantes asintomáticas como las linfadenopatías (16).

\section{MÉTODOS DIAGNÓSTICOS}

Los métodos más comunes que se usan para el diagnóstico de sialolitiasis son la sialografía convencional, la ecografía, resonancia magnética nuclear, tomografía asistida por computador (TAC), la endoscopia, en algunos casos las radiografías laterales de cráneo para sialolitos de glándula parótida y radiografías oclusales (17) (fig. 2). Estas técnicas imagenológicas denotan estructuras radiopacas circunscritas de ubicaciones variables. Estos métodos diagnósticos varían según las necesidades del paciente, según la localización del sialolito, el tamaño del mismo y los signos y síntomas que presente (18).

La obtención de imágenes diagnósticas para identificar la causa y la recurrencia de Sialolitiasis en glándulas submandibulares y sublinguales se basó en los procedimientos de radiografías convencionales (19), sialografías o radiografías oclusales usando medios de contraste cuya evidencia de sialolitiasis es poca debido a que algunos se encuentran poco calcificados o también llamados sialolitos blandos y ultrasonografía o ecografía, también útiles para el diagnostico de sialolitos renales (20). La sialografía puede

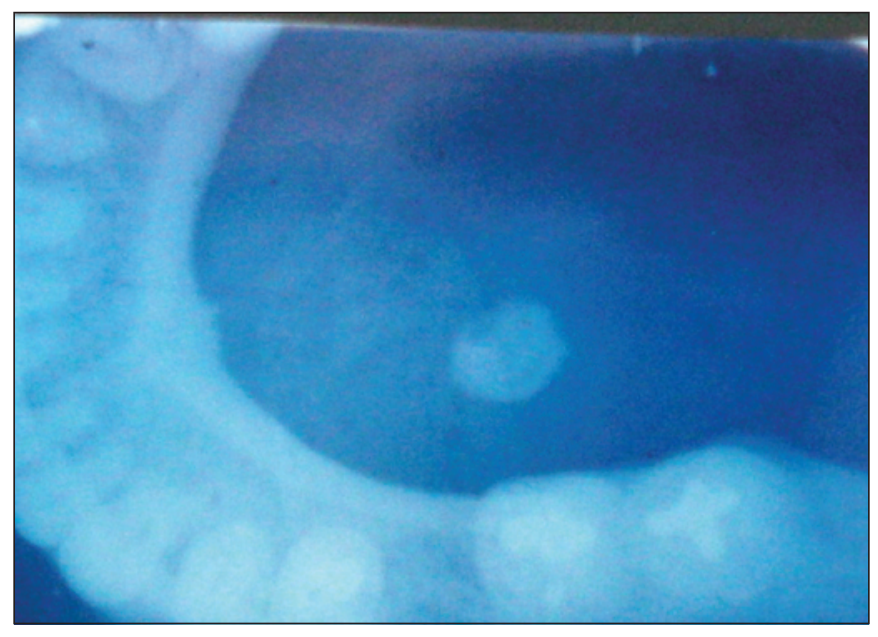

Fig. 2. Radiografía oclusal que da la imagen de un sialolito en glándula sublingual. demostrar el obstáculo como un defecto de oclusión en el conducto o estenosis; sin embargo no puede hacer una demostración de la localización del mismo. La ultrasonografía demuestra el cálculo como una estructura enérgicamente calcificada disminuyendo a nivel de la glándula el eco emitido por el ecógrafo, es uno de los métodos diagnósticos imagenológicos más eficaces (21).

El endoscopio tiene limitaciones en cuanto a su uso para las glándulas sublingual y submandibular, debido a que su alcance esta solamente para la curva del conducto de Wharton y solo es ideal para el diagnóstico y detección exacta de la causa del obstáculo, no para el tratamiento (21). Clínicamente es probable diagnosticar mediante palpación profunda de las glándulas y estructuras anexas y medición de la funcionalidad mediante técnicas exploratorias semiológicas como ordeñamiento y verificación visual de la cantidad, consistencia y color del fluido salival $(22,23)$.

\section{HALLAZGOS HISTOLÓGICOS}

Al microscopio de luz, el sialolito presenta láminas alternadas de material mineralizado de diferentes tonos y densidades alrededor de un núcleo mineralizado. En la superficie de muchos cálculos se observan fibras de colágena y células epiteliales metaplásicas, dichas estructuras posibilitan el proceso de crecimiento y mineralización activa (24).

Existen reportes que indican la presencia de una pequeña cubierta de epitelio escamoso estratificado no queratinizado acantolítico y en la porción central del sialolito, una zona formada por matriz orgánica que no está completamente mineralizada $(17,25)$.

\section{TRATAMIENTO}

Existen terapéuticas que incluyen, drenaje de la glándula afectada y en las obstrucciones profundas, cuando existe infección, ésta debe ser controlada en primer lugar con antibióticos. En algunas ocasiones se puede intentar la remoción por endoscopia; estas dos últimas técnicas reducen los riesgos de dañar a los nervios facial y lingual según sea la localización del sialolito. Se demostró que los cálculos mayores 
de $2 \mathrm{~mm}$ de diámetro son los causantes de que se presenten síntomas de sialolitiasis, además también como tratamiento se recomienda el uso de láser de $\mathrm{CO}_{2}$. La eliminación quirúrgica del cálculo (o incluso de la glándula) ha sido la alternativa clásica al tratamiento, cuando éste no es viable o no obtiene resultados. La supresión quirúrgica tiene el inconveniente según la zona en que se localice el cálculo, de poner en riesgo el nervio facial en el caso de la glándula parótida, La posibilidad de provocar una cicatriz más o menos antiestética, así como la idoneidad de abordajes intraorales contraindican el uso de técnicas quirúrgicas de abordaje extraoral (25) (Fig. 3).

Para evitar esto se describió recientemente una técnica consistente en la fragmentación del cálculo mediante ondas expansivas ultrasónicas que no requiere anestesia, sedación ni analgesia. El procedimiento dura unos 30 minutos y se realizan sesiones sucesivas semanalmente hasta la total eliminación de los fragmentos del cálculo ayudado por sialogogos (26). Esta litotricia extracorpórea fragmenta los cálculos menores de $2 \mathrm{~mm}$ de diámetro, evita la necesidad de la cirugía e incluso la administración de anestesia o sedantes (27).

Este cuadro clínico se puede manejar con procedimientos no quirúrgicos si son pequeños como procedimientos conservadores, mediante la infusión de espasmolíticos, penicilina y o suero fisiológico en el interior del conducto. Afirman que esta aplicación tópica es mucho más efectiva, debido a la poca recidiva del proceso, que la administración sistémica.

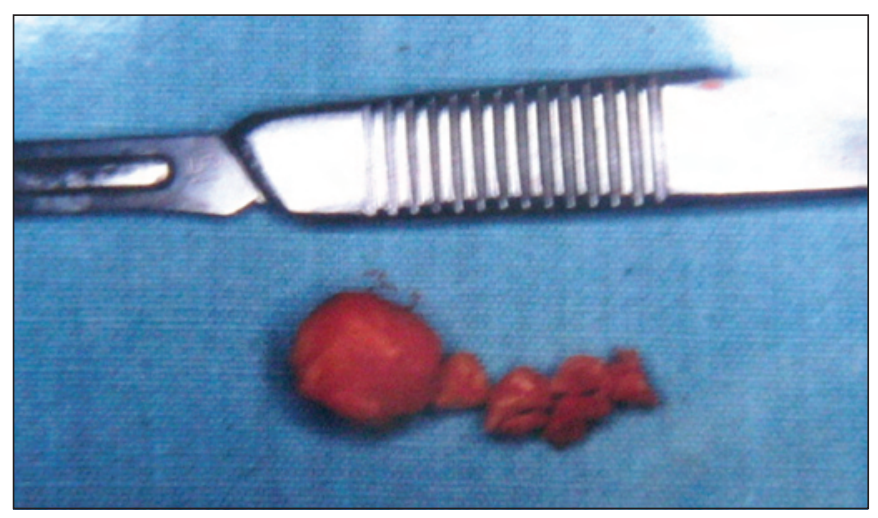

Fig. 3. Imagen macroscópica de un sialolito postextracción quirúrgica.
Este tratamiento presenta como ventaja la actuación a distintos niveles: dilata el conducto, libera los sialolitos adheridos y enjuaga la albúmina coagulada que obstruye el conducto $(28,29)$.

Después de la eliminación de los cálculos salivales se debe realizar estimulación continua con sialogogos, los más utilizados son el jugo de limón, el ácido ascórbico y la goma de mascar. Sin embargo, un estudio con esta técnica se realizó en un grupo de pacientes que sufrieron de sialolitiasis, demostró que el $75 \%$ de las glándulas afectadas funcionan normalmente después de la remoción del cálculo (30).

Estas lesiones pueden presentar recidivas o en caso de que haya persistencia de la obstrucción puede causar la destrucción intensa del componente parenquimatoso de la glándula y provocar una sialoadenitis crónica irreversible que va a exigir la eliminación de la glándula (9).

\section{CONCLUSIÓN}

La obstrucción de las glándulas salivares mayores y menores por la presencia de un sialolito representa una entidad patológica multifactorial, así como la variabilidad que hay entre sus métodos de diagnostico y tratamiento. La sialolitiasis afecta a la población adulta masculina en una mayor incidencia obteniéndose como primeras manifestaciones clínicas la hernia de la glándula de manera espontánea o bien al momento de consumir alimentos, suele ser en la mayoría de los casos asintomática y de carácter sintomático cuando presenta un tamaño considerable. Clínicamente es importante diferenciar esta patología de otras alteraciones glandulares debido a que sus signos y síntomas se relacionan con otros agrandamientos glandulares. Imagenológicamente se proponen diversos métodos para la evidencia radiográfica, pero esto es dependiente del carácter del sialolito, ya sea en tamaño, localización del mismo (debido a que no necesariamente se puede encontrar en el trayecto y/o desembocadura de los conductos excretores de determinada glándula salivar sino en el cuerpo de la misma), signos y síntomas, necesidad del especialista para proveer un acertado diagnostico. En cuanto al tratamiento y pronostico también son variables ya sea primordialmente la ubi- 
cación del sialolito, la glándula implicada, el tamaño grado de mineralización y la necesidad o no de extirpar la glándula.

Las ayudas por imagen suelen ser quienes determinan el carácter de la enfermedad entre ellas se usan las sialografías como radiografías oclusales usando medios de contraste, las ecografías recomendadas ya que dan una localización y diagnostico certero de sialolitiasis, la endoscopia como método de diagnostico es de utilidad debido a que provee al operador la visión indirecta por medio de un monitor, el trayecto del conducto, tamaño y localización del material calcificado sobre todo para la glándula parótida, otras ayudas imagenológicas también son útiles como la radiografía lateral de cráneo con medio de contraste para el diagnostico de sialolitiasis de glándula parótida, tomografía computarizada y la resonancia magnética nuclear. Para el tratamiento de sialolitiasis se usan métodos quirúrgicos y no quirúrgicos desde el uso de espasmolíticos, antibióticos (si es de carácter bacteriano), terapia física, sialogogos, líquido abundante, analgésicos hasta extirpación quirúrgica que en algunos casos conlleva a la eliminación de la glándula. Todos estos métodos diagnósticos y terapéuticos dependen directamente de la naturaleza de la lesión, ubicación, composición, consistencia, tamaño y aspectos propios del paciente.

\section{BIBLIOGRAFÍA}

1. El Deeb M, Holte N, Gorlin RJ. Submandibular salivary gland sialoliths perforated through the oral floor. Oral Surg Oral Med Oral Pathol. 1981 Feb;51(2):134-9.

2. Lustmann J, Regev E, Melamed Y. Sialolithiasis. A survey on 245 patients and a review of the literature. Int J Oral Maxillofac Surg. 1990 Jun;19(3):135-8.

3. Orlian AI, Schaefer M, Golub J. Multiple bilateral sialoliths of the submandibular ducts. N Y State Dent J. 1998 Aug-Sep;64(7):42-3.

4. Bodner L. Parotid Sialolithiasis. J Laryngol Otol. 1999 Mar;113(3):266-7.
5. Harrison JD, Epivatianos A, Bhatia SN. Role of microliths in the aetiology of chronic submandibular sialadenitis: a clinicopathological investigation of 154 cases. Histopathology. 1997 Sep;31(3):237-51.

6. Marchal F, Kurt AM, Dulguerov P, Lehmann W. Retrograde theory in sialolithiasis formation. Arch Otolaryngol Head Neck Surg. 2001 Jan;127(1): 66-8.

7. Nahlieli O, Eliav E, Hasson O, Zagury A, Baruchin AM. Pediatric sialolithiasis. Oral Surg Oral Med Oral Pathol Oral Radiol Endod. 2000 Dec;90 (6):709-12.

8. Paterson JR, Murphy MJ. Bones, groans, moans... and salivary stones? J Clin Pathol. 2001 May;54(5):412.

9. Bodner L. Giant salivary gland calculi: diagnostic imaging and surgical management. Oral Surg Oral Med Oral Pathol Oral Radiol Endod. 2002 Sep;94(3):320-3.

10. Roh JL. Removal of the submandibular gland by a submental approach: a prospective, randomized, controlled study. Oral Oncol. 2008 Mar;44(3): 295-300.

11. Marchal F, Dulguerov P. Sialolithiasis management: the state of the art. Arch Otolaryngol Head Neck Surg. 2003 Sep;129(9): 951-6.

12. Pons Vicente O, Almendros Marqués N, Berini Aytés L, Gay Escoda C. Minor salivary gland tumors: A clinicopathological study of 18 cases. Med Oral Patol Oral Cir Bucal. 2008 Sep 1;13(9):E582-8.

13. Lomas DJ, Carroll NR, Johnson G, Antoun NM, Freer CE. MR sialography. Work in progress. Radiology. 1996 Jul;200(1):129-33.

14. Fox RI, Liu AY. Sjögren's syndrome in dermatology. Clin Dermatol. 2006 Sep-Oct;24 (5):393-413.

15. Jordan RC, Speight PM. Lymphoma in Sjögren's syndrome. From histopathology to molecular 
pathology. Oral Surg Oral Med Oral Pathol Oral Radiol Endod. 1996 Mar;81(3):308-20.

16. Pardal Refoyo JL, Muñoz Herrera A, Criado Martín D. [The development of the pleomorphic adenoma in the accessory salivary gland and surgical approach by endobucal techniques]. Acta Otorrinolaringol Esp. 1993 Sep-Oct;44(5): 395-7.

17. Becker M, Marchal F, Becker CD, Dulguerov P, Georgakopoulos G, Lehmann W, Terrier F. Sialolithiasis and salivary ductal stenosis: diagnostic accuracy of MR sialography with a three-dimensional extended-phase conjugatesymmetry rapid spin-echo sequence. Radiology. 2000 Nov;217(2):347-58.

18. van den Akker HP, Busemann-Sokole E. Submandibular gland function following transoral sialolithectomy. Oral Surg Oral Med Oral Pathol. 1983 Oct;56(4):351-6.

19. Moskow R, Moskow B, Robinson H. Minor salivary gland sialolithiasis. Oral Surg, Oral Med, Oral Pathol, 1964;17:225-7.

20. Nguyen BD. Demonstration of renal lithiasis on technetium-99m MDP bone scintigraphy. Clin Nucl Med. 2000 May;25(5):380-2.

21. Chu DW, Chow TL, Lim BH, Kwok SP. Endoscopic management of submandibular sialolithiasis. Surg Endosc. 2003 Jun;17(6):876-9. Epub 2003 Mar 7.

22 Zenk J, Koch M, Bozzato A, Iro H. Sialoscopyinitial experiences with a new endoscope. $\mathrm{Br} J$ Oral Maxillofac Surg. 2004 Aug;42(4):293-8.

23. Königsberger R, Feyh J, Goetz A, Kastenbauer E. Endoscopically-controlled electrohydraulic intracorporeal shock wave lithotripsy (EISL) of salivary stones. J Otolaryngol. 1993 Feb;22(1): 12-3.
24. Shigeishi H, Hayashi K, Takata T, Kuniyasu H, Ishikawa T, Yasui W. Pleomorphic adenoma of the parotid gland with extensive bone formation. Pathol Int. 2001 Nov;51(11):883-6.

25. Dulguerov P, Marchal F, Lehmann W. Postparotidectomy facial nerve paralysis: possible etiologic factors and results with routine facial nerve monitoring. Laryngoscope. 1999 May;109 (5):754-62.

26. Schlegel N, Brette MD, Cussenot I, Monteil JP. [Extracorporeal lithotripsy in the treatment of salivary lithiasis. A prospective study apropos of 27 cases] Ann Otolaryngol Chir Cervicofac. 2001 Dec;118(6):373-7.

27. Capaccio P, Ottaviani F, Manzo R, Schindler A, Cesana B. Extracorporeal lithotripsy for salivary calculi: a long-term clinical experience. Laryngoscope. 2004 Jun;114(6):1069-73.

28. Antoniades D, Harrison JD, Epivatianos A, Papanayotou P. Treatment of chronic sialadenitis by intraductal penicillin or saline. J Oral Maxillofac Surg. 2004 Apr;62(4):431-4.

29. Steinberg MJ, Herréra AF. Management of parotid duct injuries. Oral Surg Oral Med Oral Pathol Oral Radiol Endod. 2005 Feb;99(2):136-41.

30. Ottaviani F, Galli A, Lucia MB, Ventura G. Bilateral parotid sialolithiasis in a patient with acquired immunodeficiency syndrome and immunoglobulin G multiple myeloma. Oral Surg Oral Med Oral Pathol Oral Radiol Endod. 1997 May;83(5):552-4.

\section{CORRESPONDENCIA}

Antonio Díaz Caballero

Campus de la Salud. Barrio Zaragocilla

Cartagena. D.T y C. Colombia.

Correo electrónico: antoniodiazc@yahoo.com. 